\title{
Minimization procedure of experimental tests for calibration purposes, within HVAC\&R energy efficiency framework
}

\author{
S. Morales-Ruiz ${ }^{1}$, C. Oliet ${ }^{l}$, J. Castro $^{l}$, J. Rigola $^{l}$, A. Oliva $^{l}$ \\ ${ }^{I}$ Technical University of Catalonia-BarcelonaTech, CTTC, \\ C/ Colom, 11, Terrassa, E-08222, Spain, \\ +34937398736,cttc@cttc.upc.edu
}

\begin{abstract}
Simulation models and predictive tools need to be fast, accurate and robust at the same time. The models that have to provide numerical solutions under transient conditions for a long period of time need to be simple with the aim of minimizing the time respond, without losing the accuracy. Thus, previous experimental data and a calibration methodology are necessary to assure this objective, both are strictly necessary to reproduce the behaviour with accuracy expected. Consequently, even accurate information (e.g. look-up tables) for HVAC\&R components (e.g. heat exchangers, fan/compressor, auxiliary elements, etc.) is known and all coupling system is developed, the minimization of experimental tests for calibration purposed based scattered data interpolation is now an important aspect, which looks for reducing the quantity of experiments necessary to assure the accuracy expected from an optimization point of view. The present work shows an optimization procedure based on test number minimization according detailed error comparison against existing previous data. Illustrative results for a specific component are presented highlighting test number reduction without losing accuracy.
\end{abstract}

\section{Introduction}

Energy Efficiency in Buildings, can be mainly based on: systems improvements (i.e. better HVAC\&R components and systems); energy consumption reduction (i.e. retrofitting solutions), renewable energy use (i.e. net-zero building), and control strategies that couple energy performance in building behaviour. In that sense, accurate numerical simulation tools are very important to analyse the behaviour of any kind of component or system looking for the energy efficiency of it.

A wanted simulation tool needs to be fast, accurate and robust, all at the same time. Then, a reduced time respond, without losing the accuracy is an important characteristic in any kind of numerical simulation model. Using previous information of the component or the system into the numerical model is a way to develop a fast and accurate simulation tool. Many times the previous information is organized in an array or matrix of data, which is known as look-up table.

The look-up tables are used in different modern engineering applications where a high number of parameters are required to characterize a phenomenon. In this case, the look-up tables are a good alternative to reduce the complexity of the calculation. Also, an advantage of using look-up tables into the numerical model is the ease of modifying data by changing the height or value according to the node [1].

A calibration methodology is required to develop and configure an accurate numerical simulation tool. In other words, the success of the application of any numerical model is strongly dependent on how precisely the model is calibrated [2].

In general, numerical approaches are not as accurate as black-box models. There are errors due to many different factors or sources, for example, incomplete information of the system properties, consider different assumption, apply simplified representations of complex physical processes, and other uncontrollable factors [3].

The numerical simulation tool has to be calibrated against a set of measurements (experimental data) with the aim of obtaining accurate numerical results that represents the real working condition of the component, system or unit simulated.

The calibration methodology is based on identifying a general rule, which defines a phenomenon or process behaviour, and compare it with the numerical results obtained. Defining a general rule is not an easy task, because the rule is evaluated from a set of data that has

Corresponding author: cttc@,cttc.upc.edu 
been obtained in a limited location or conditions. The real difficulty is to extend this rule to other location or range conditions in which the measurements were obtained. Thus, the effort consist in finding a rule or function $P_{f}$ that fit very well in the measured range but gives good fit in points outside of the measured range. A way to achieve this objective is using any kind of interpolation method [4].

In this paper, a calibration methodology is proposed to recalibrate the numerical model of a Heat eXchanger (HX). Besides of this, a procedure to define the minimum number of experimental points required to recalibrate the model is described. This methodology can be applied to different HVAC\&R components: fan, evaporator, condenser, expansion valves, etc.

\section{Calibration methodology of the model}

The strategy followed to calibrate a numerical model is the comparison between experimental data ( $\mathrm{Y}_{\text {MEASURED }}$ ) and the numerical results obtained with the model $\left(\mathrm{Y}_{\text {MODEL }}\right)$. A corrector value $\left(\mathrm{E}_{\text {CORRECTOR }}\right)$ is obtained from the comparison, which is a new input in the numerical model. The corrector value will help to modify the original numerical model with the aim of finding an accurate numerical result that represents the real behaviour of the component or system simulated. A blocks diagram with the calibration method is shown in Figure 1.

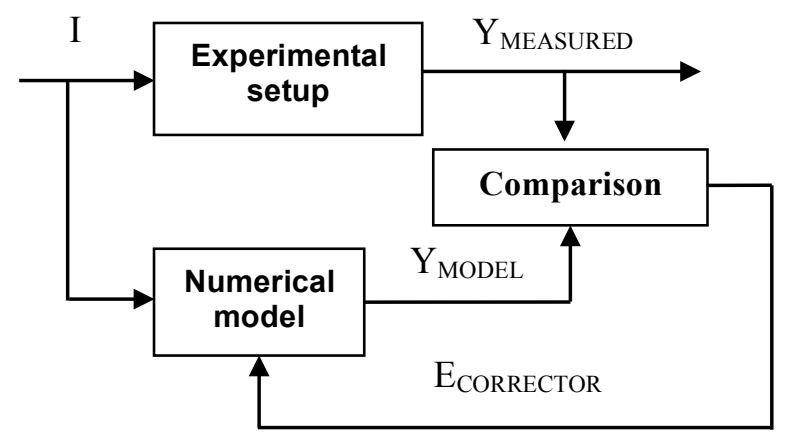

Fig. 1. Block diagram of the calibration.

A look-up table correction methodology will be explained and applied on one HVAC\&R component, in this case the HX. A schematic representation of the component is depicted in Figure 2, where the mass flows are expressed as Q, whilst the temperatures are identified as $\mathrm{T}$.

The calibration method consists of modifying the original look-up table (previously known) to a new lookup-table that considers integration effects. To achieve this objective the next look-up table correction strategy is proposed and applied on the HX efficiency:

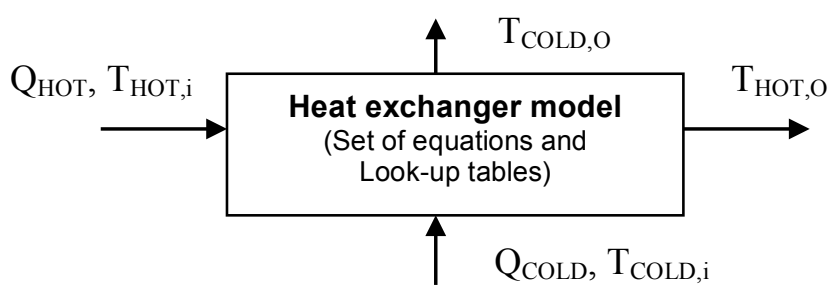

Fig. 2. Schematic representation of the heat exchanger (HX).

- Generating a look-up-table with corrector values "Eff corrector", which takes old efficiency and obtains a new efficiency, therefore acquiring all previous information as the baseline values:

$$
E f f_{\text {new }}(i, j)=\operatorname{Eff}_{\text {old }}(i, j) * E f f_{\text {corrector }}(i, j)
$$

- Scattered values of this corrector will be obtained from experiments at certain flow values $(i, j)$, in this specific case mass flow of the hot current $\mathrm{Q}_{\text {нот }}$ and mass flow of the cold current $\mathrm{Q}_{\mathrm{COLD}}$ :

$$
E f f_{\text {corrector }}=\text { Integrated }_{\text {eff }} / \text { Isolated }_{\text {eff }}
$$

- Applying an interpolation-extrapolation method to obtain the Eff $f_{\text {corrector }}$ for points without experimental information from the scattered values previously evaluated.

An important aspect is evaluating the minimum number of experimental points required to applied the strategy presented looking for obtaining accuracy results.

\subsection{Interpolation methods}

Different interpolation/extrapolation methods or MATLAB functions can be used to obtain Eff corrector values for all points with and without experimental information; some of them are mentioned and shortly described:

- Scattered Interpolation: This method is used when the measurement location is not distributed in a regular grid (scattered data). The scatteredInterpolant function returns the interpolant $F$ for the given data set. You can evaluate $F$ at a set of query points, such as $(x q, y q)$ in 2-D, with the aim of producing interpolated values $V q=$ $F(x q, y q)$.

- $\quad$ Fit function: Using the algorithm options specified by the fitOptions object of the fit function, a fit to the data could be created. Different kind of fit type can be used (e.g.: least-squares, linear polynomial surface, linear interpolation, quadratic interpolation, piecewise cubic interpolation, local linear regression, local quadratic regression). Two fit types are described:

- Least squares: This method proves the parameter values for the model that defining the best fits of the data. A model function $F(x, \boldsymbol{B})$ is 
defined, where $m$ parameters are held in the vector $\mathbf{B}$. The result of the fitting process is an estimate of the model parameters, together with the sum of the squares of the residuals.

- Polynomial estimation: Returns the coefficients for a polynomial $p(x)$ of degree $n$ that is a best fit (in a least-squares sense) for the data in $y$. The coefficients in $p$ are in descending powers, and the length of $p$ is $n+1$ :

$p(x)=p_{1} x^{n}+p_{2} x^{n-1}+\cdots p_{n} x+p_{n+1}$
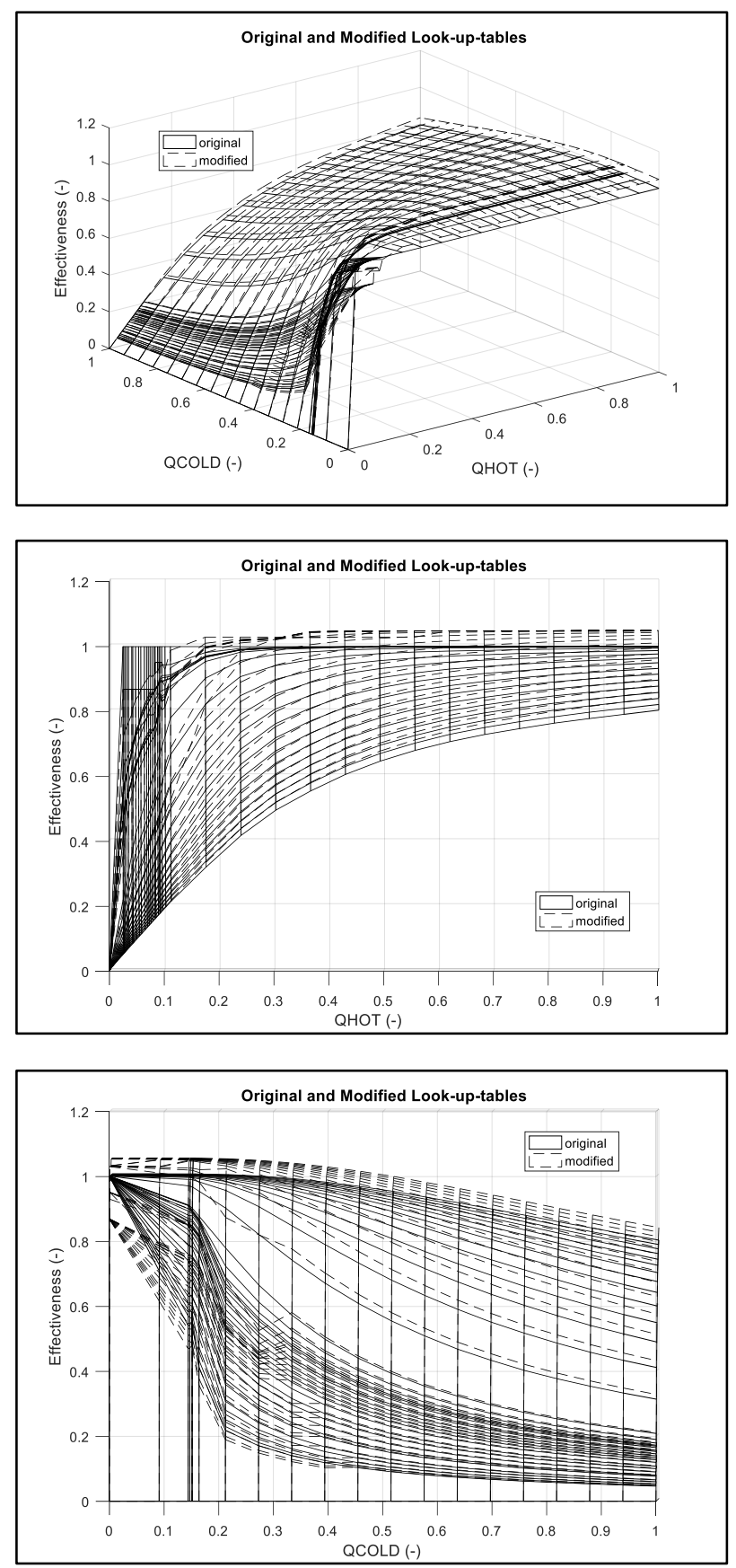

Fig. 3. Original and modified Look-up table comparison (top); Effectiveness vs. $\mathrm{Q}_{\text {Hот }}$ (middle); and Effectiveness vs. Q $\mathrm{COLD}$ (bottom)

\subsection{Calibration of the HX effectiveness look-up table}

Applying the strategy proposed in the last section, the look-up table of the HX effectiveness has been analysed.

The original look-up table (previously known) and modified look-up table (generated from experimental data and after applying the calibration methodology) of the effectiveness are depicted in Figure 3. The comparison between both effectiveness values (original and modified) is represented in function of the mass flows $\left(\mathrm{Q}_{\mathrm{HOT}}\right.$ and $\left.\mathrm{Q}_{\mathrm{COLD}}\right)$ which are expressed in dimensionless form.

The scattered values obtained from experimental data are shown in Figure 4, together with the ratio between the original and the modified look-up tables. The Eff corrector values obtained have given as result that more experimental data are needed to cover better the operating range of the HX. Then, a strategy to define exactly the perimeter operating range and the minimum number of points required to carry out an efficient and complete calibration of the HX will be presented.
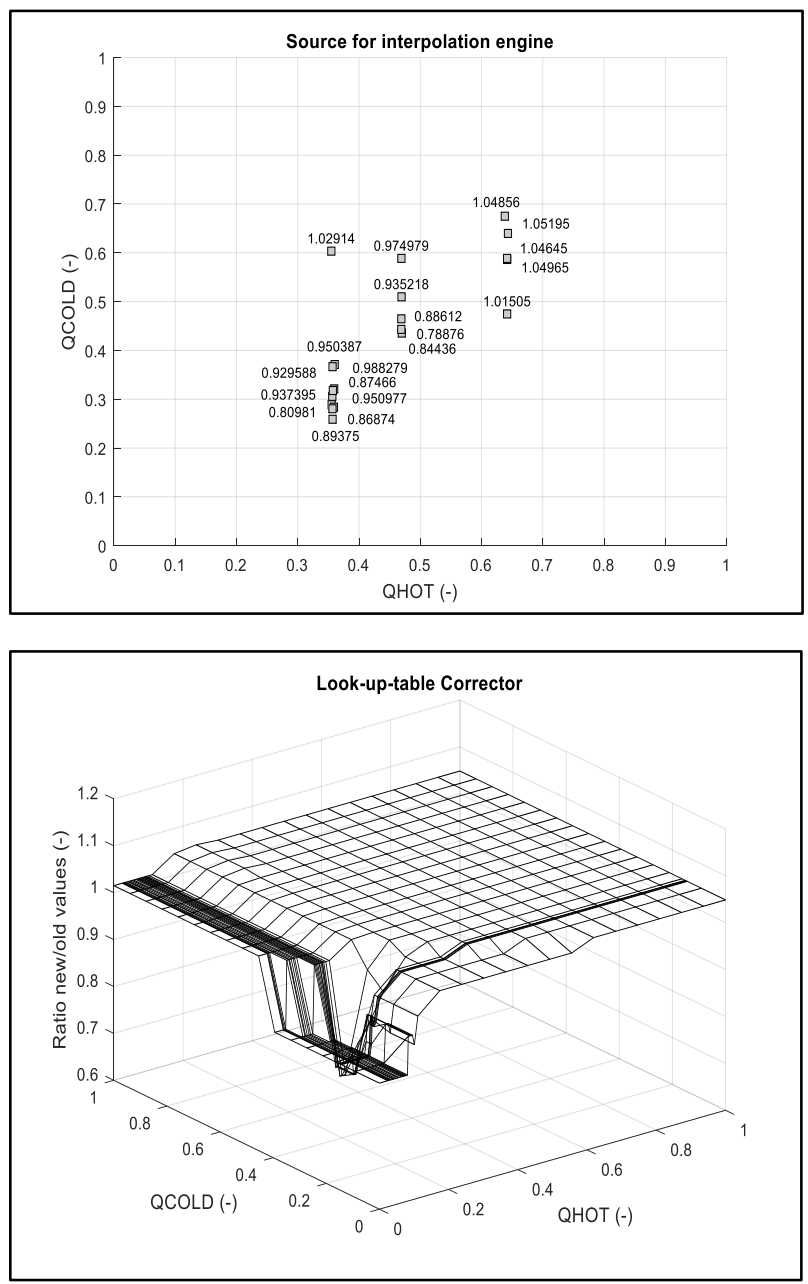

Fig. 4. Source for interpolation-scattered values (top) and the Eff corrector result (bottom). 


\section{Test points optimization}

In this section an optimization procedure based on test number minimization will be defined to achieve the calibration of a component (HX). A virtual tool, which includes the device and the system constraints, is used to define the number of points to be tested.

The group of working points should be into an operating range (previously defined in function of the constraints) configuring a matrix of points. Now, the valid perimeter of the matrix of points and the minimum number of points will be evaluated in function of different criteria.

\subsection{Valid points to be tested}

First of all, the HX operating range will be well defined. The Hot and Cold mass flows and the fluids temperature at the inlet ranges, together with the temperature values allowed at the outlet of the HX should be reviewed and established.
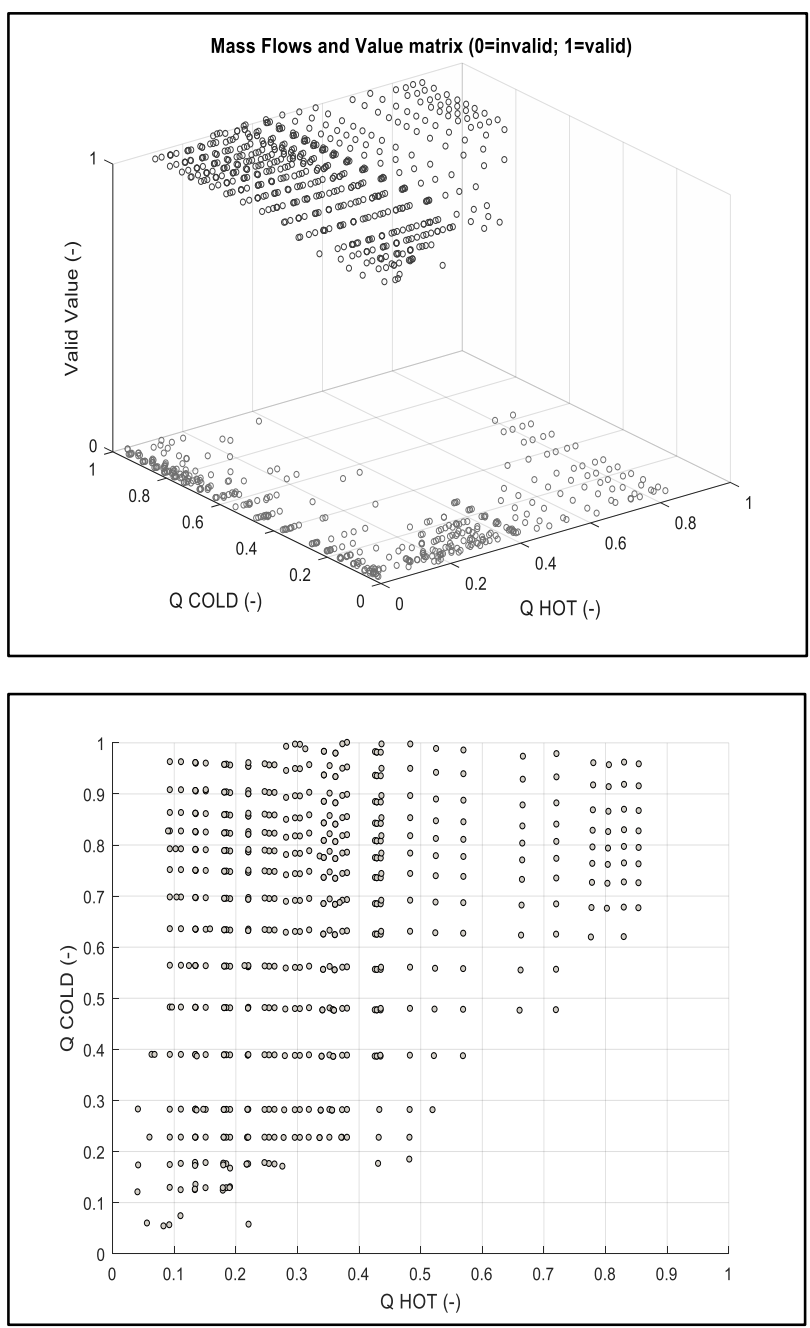

Fig. 5. Matrix of points with valid and invalid points (top) and matrix of point with valid points to be tested (bottom).

After that, a matrix of possible points, to be tested experimentally, will be proposed. Applying some criteria to find the valid points in function of the operating range (safety working conditions of the component and the system) two different kinds of points are defined. Points with value equal to 1 mean valid points to test, whilst points with value equal to 0 mean invalid points. The invalid points will be out of the test. The matrix point with valid and invalids points are depicted in Figure 5, together with a second graphical representation that shows the final valid points.

\subsection{The perimeter operating range}

The group of points classified as valid has been used to define a contour or perimeter, which can be evaluated from any set of scattered values [5]. This contour has been called as "perimeter operating range".

All the points that are inside the perimeter operating range are considered as possible points to be tested, while the others points are rejected.

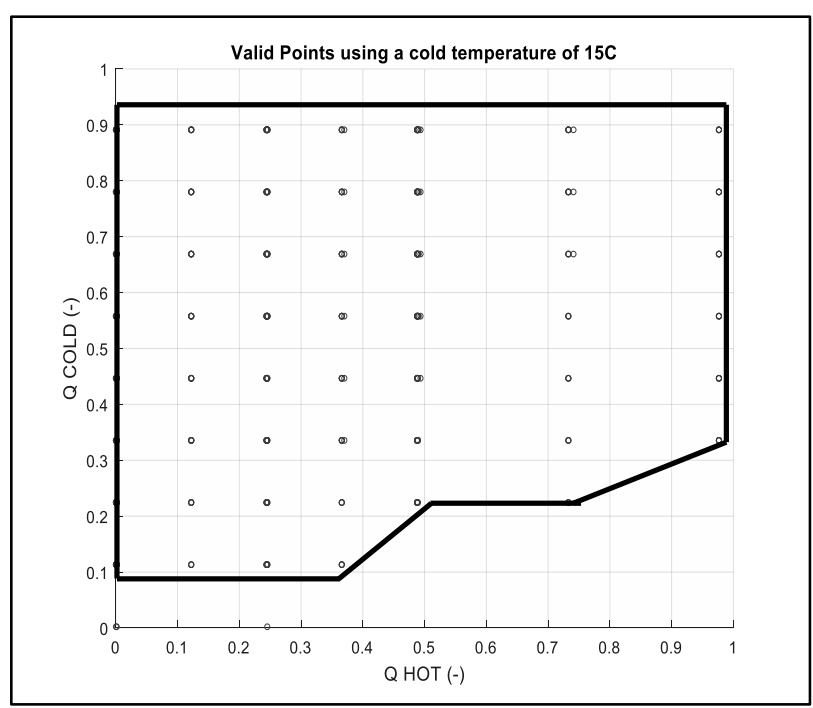

Fig. 6. Perimeters in function of the cold fluid flow temperature at the inlet $\left(15^{\circ} \mathrm{C}\right)$.

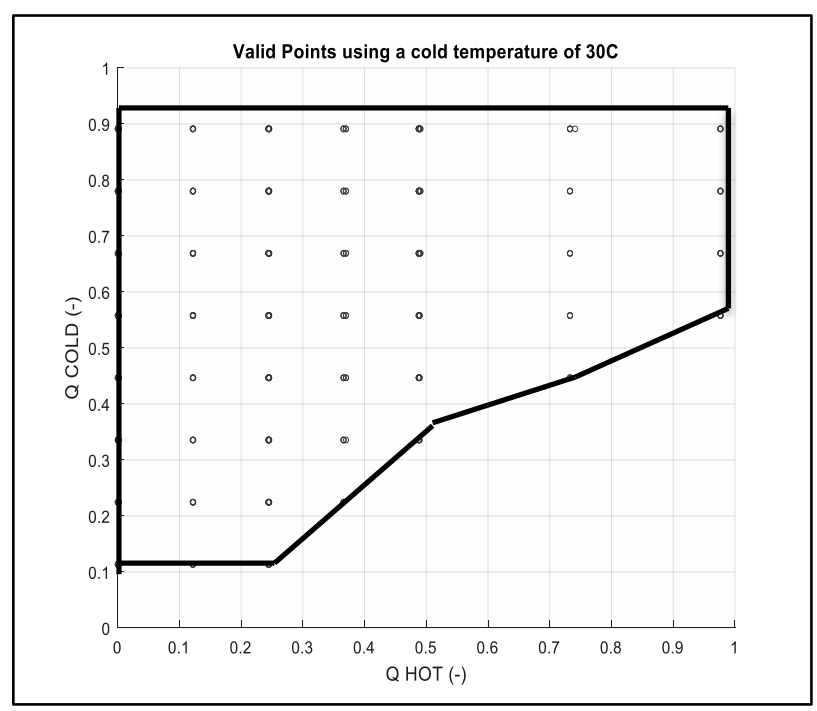

Fig. 7. Perimeters in function of the cold fluid flow temperature at the inlet $\left(30^{\circ} \mathrm{C}\right)$. 


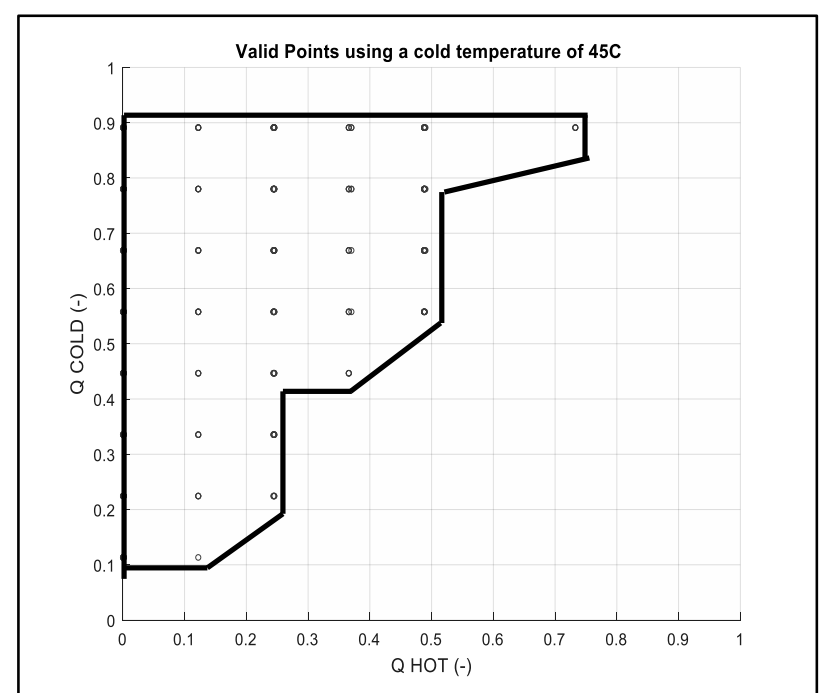

Fig. 8. Perimeters in function of the cold fluid flow temperature at the inlet $\left(45^{\circ} \mathrm{C}\right)$.

The shape of the perimeter operating range can be affected by different conditions. The inlet temperature of the cold current $\left(\mathrm{T}_{\mathrm{COLD}, \mathrm{i}}\right)$ has been chosen to analyse the effect of this value on the perimeter operating range of the HX device. The influence of this parameter on the perimeter shape is shown in Figures 6, 7 and 8 in which a reduced perimeter operating range is obtained when the inlet temperature is increased. This effect occurs because any variation in the inlet temperature of the cold flow (increasing it) affect the outlet temperatures of the hot and cold fluid flows, one of them or both values could be conditioned by the operating range, giving as result that the perimeter operating range should be reduced.

\subsection{The location of the point into the perimeter}

Once the perimeter operating range has been defined, a number of points and the location of these points into the perimeter will be carried out.

The proposal consists of using the variation of the variable defined by the look-up table in function of the dependent parameters to obtain the module gradient, i.e.: evaluating the variation of the Effectiveness (Eff) in function of the hot mass flow $\mathrm{Q}_{\text {нот }}$ and the cold mass flow $\mathrm{Q}_{\mathrm{COLD}}\left(\frac{d E f f}{d Q_{H O T}}\right.$ and $\frac{d E f f}{d Q_{C O L D}}$, respectively).

The highest variation values are used as criterion to propose an intensification of the points in that zone. In this case an intensification zone is proposed for lower mass flows values of both currents $\left(\mathrm{Q}_{\text {HOT }}\right.$ and $\left.\mathrm{Q}_{\mathrm{COLD}}\right)$. This zone is marked into a rectangle in dash line and grey colour as can be seen in Figure 9 .

Regarding to the distribution of points into the perimeter, two different ways could be used: a regular or a random distribution of points. A graphical representation of both alternatives is shown in Figure 10.
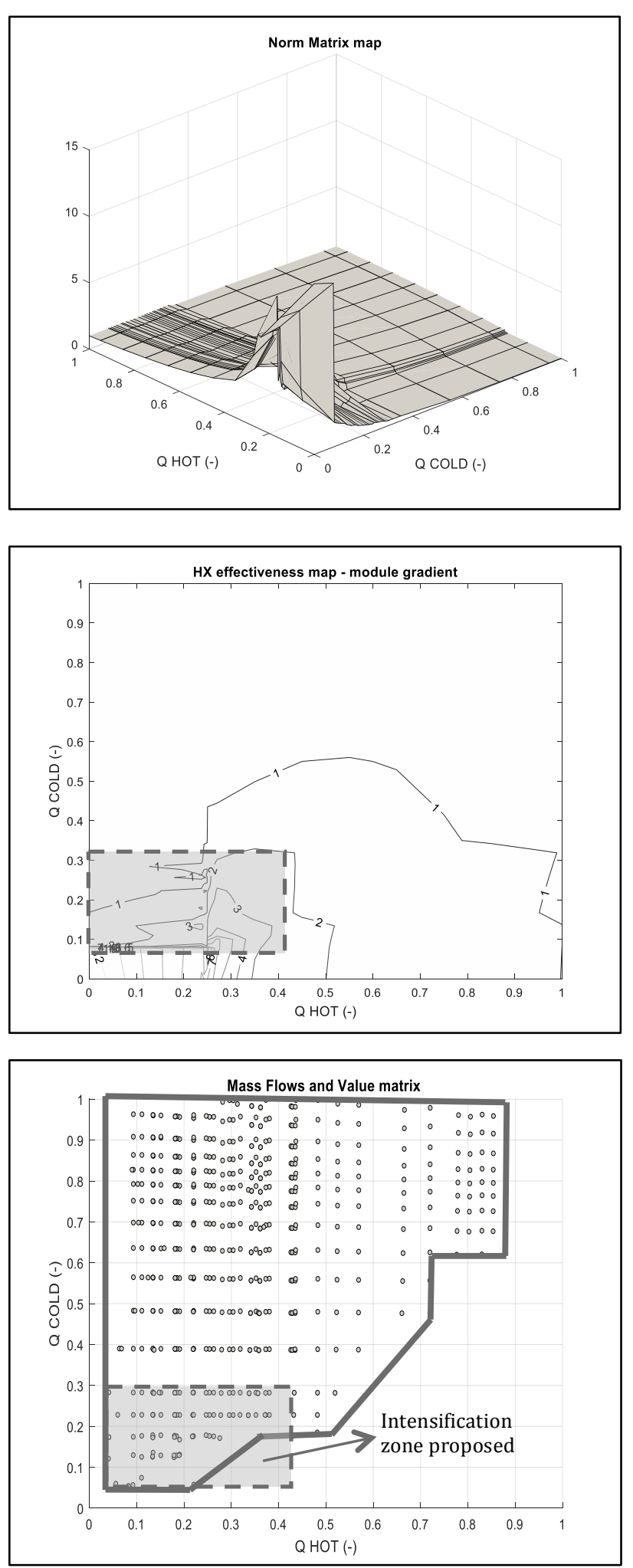

Fig. 9. Module gradient of effectiveness, perimeter range and intensification zone.

Probably a regular or uniform distribution of points within the perimeter will result in a better representation of the variable studied than a random or non-uniform distribution. 

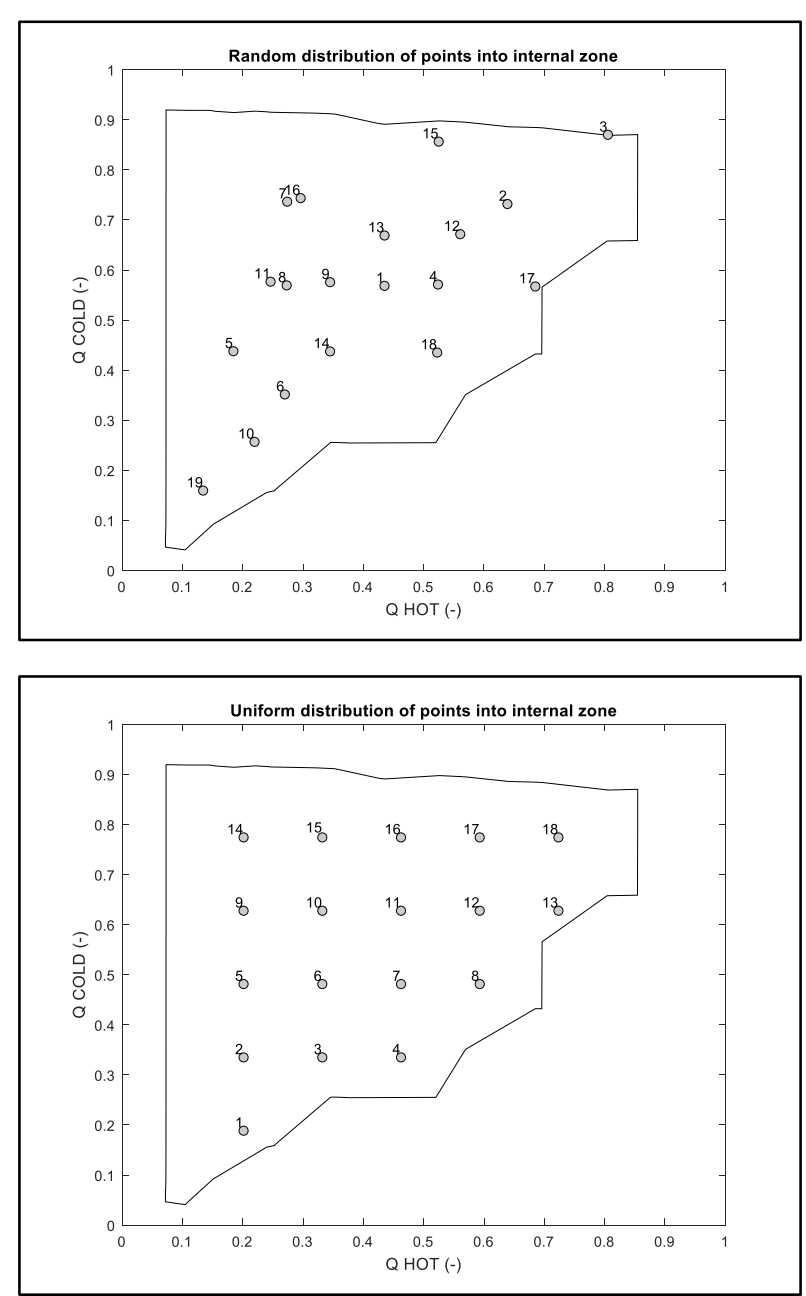

Fig. 10. Random and uniform distribution of points into the perimeter.

\subsection{The minimum number of points}

The next step is defining the minimum number of points to be tested. The criterion followed to define the minimum number of points is the mean relative error value, evaluated between the specific value (e.g.: the effectiveness in the HX) obtained from the original lookup table (previously known), and the value obtained from the variable interpolation (obtained after applying a fit function on the minimum number of points defined). This criterion could be used on all the components of any HVAC\&R system (HX, Condenser, Evaporator, Fan, Expansion valve, etc.) to find the most adequate matrix of points, which will be capable to define the value of a specific variable that characterize the behaviour of the component.

$$
\begin{aligned}
& \text { Difference }=\left|E f f_{\text {original }}-E f f_{\text {interpolated }}\right| \\
& \text { Error }=\frac{\left|E f f_{\text {original }}-E f f_{\text {interpolated }}\right|}{E f f_{\text {original }}} \times 100
\end{aligned}
$$

Other criteria could be used to evaluate the fit between the original and interpolated values and define as valid a number of points, some of them could be: the mean of the sum of square errors, the mean of absolute errors, or the mean of absolute percentage errors [3].
The influence of the number of points in the error obtained for the Effectiveness variable is depicted in Figure 11.

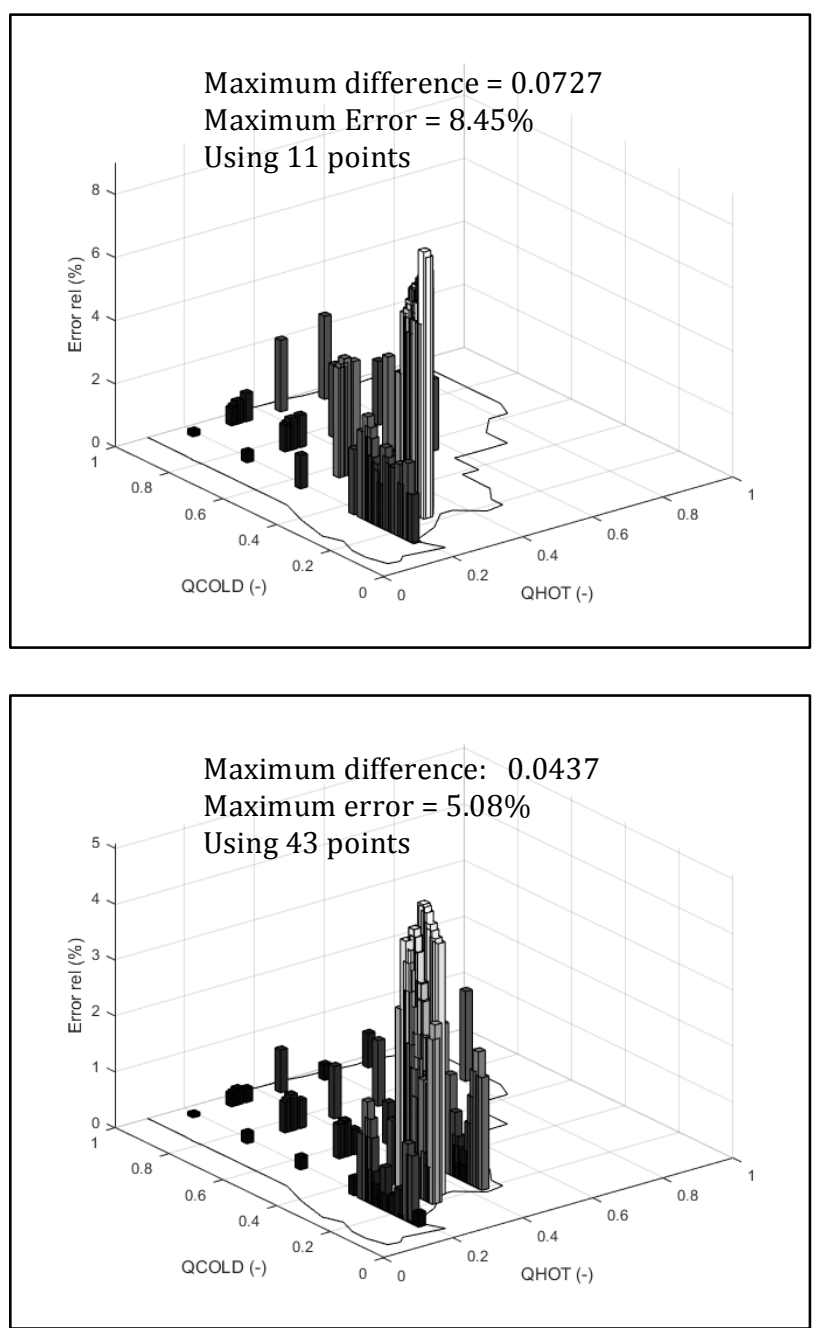

Fig. 11. The error distribution in function of the number of points, using scatteredInterpolant function.

\section{The interpolation method influence}

The influence of using the scatteredInterpolat function, the griddata function and the fit function, all from MATLAB [6], in the value interpolation has been tested.

Using the scatteredInterpolant and griddata function give as result a high value in the relative error of the variable studied (Effectiveness in the HX) as consequence of the triangulation method used by these both interpolation functions [6]. The way to avoid this problem was using the fit function, which is capable to give better results than the others because this function does not apply any triangulation method to solve the interpolation. The Effectiveness surface obtained by means of two different interpolation functions for the case of 26 points is shown in figure 12 . 

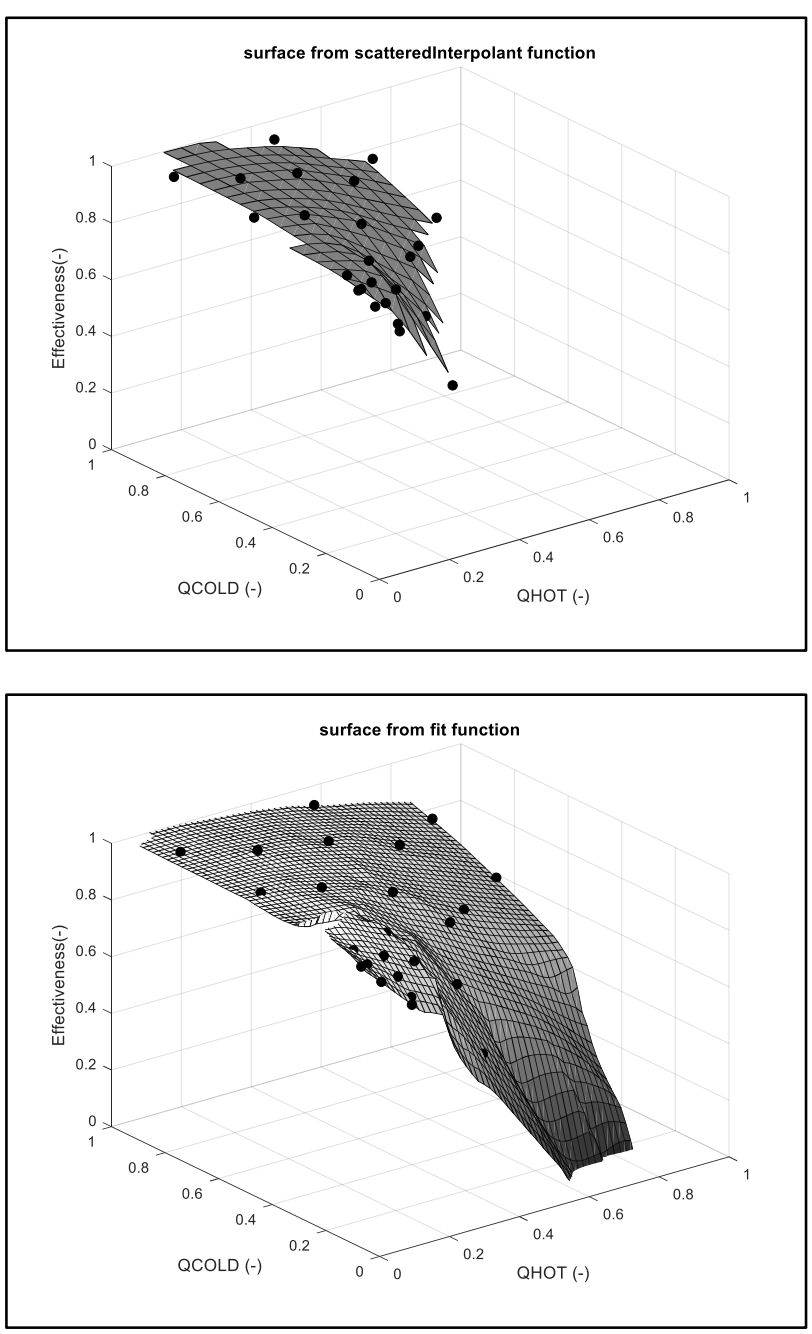

Fig. 12. Effectiveness values obtained using 26 points and two different interpolation functions.

Using the fit function and the fit type loess (Local regression using weighted linear least squares and a 2nd degree polynomial model) produces a surface that represents better the set of scattered points than the surface obtained with scatteredInterpolant function.

The error of the effectiveness value for the $\mathrm{HX}$ in function of the number of points and the interpolation method applied is presented in table 1 .

Table 1. Error in function of the interpolation method

\begin{tabular}{|l|c|c|c|c|}
\hline $\begin{array}{c}\text { Number } \\
\text { of points }\end{array}$ & \multicolumn{2}{|c|}{$\begin{array}{c}\text { scatteredInterpolant } \\
\text { Function }\end{array}$} & \multicolumn{2}{c|}{$\begin{array}{c}\text { fit (loess) } \\
\text { Function }\end{array}$} \\
\hline & $\begin{array}{c}\text { Max } \\
\text { Error } \\
(\%)\end{array}$ & $\begin{array}{c}\text { Mean } \\
\text { Error } \\
(\%)\end{array}$ & $\begin{array}{c}\text { Max } \\
\text { Error } \\
(\%)\end{array}$ & $\begin{array}{c}\text { Mean } \\
\text { Error } \\
(\%)\end{array}$ \\
\hline 11 & 8.45 & 3.94 & 9.57 & 3.68 \\
\hline 26 & 8.63 & 3.43 & 4.61 & 1.30 \\
\hline 35 & 5.21 & 2.08 & 5.69 & 1.52 \\
\hline 43 & 5.08 & 1.85 & 6.18 & 1.09 \\
\hline
\end{tabular}

\section{The optimization results}

The application of the optimization test points on the HX has been carried out and giving next result:

- A perimeter operating range is well defined by 11 points; the contour is marked by a continuous line.

- The minimum number of points proposed that define the HX effectiveness is 26 .

- The maximum error value of $4.61 \%$ and a mean error value of $1.3 \%$ are obtained when the fit function is used.

The perimeter, number of points and error are presented in function of the mass flows, which are expressed in dimensionless way, in figures 13 and 14.

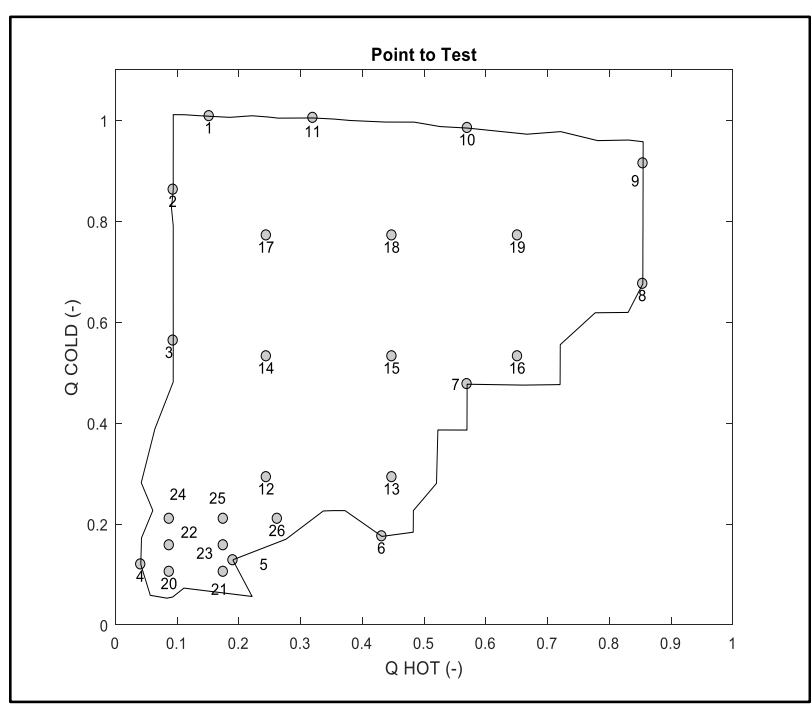

Fig. 13. Minimum number of points to be tested and the perimeter operating range.

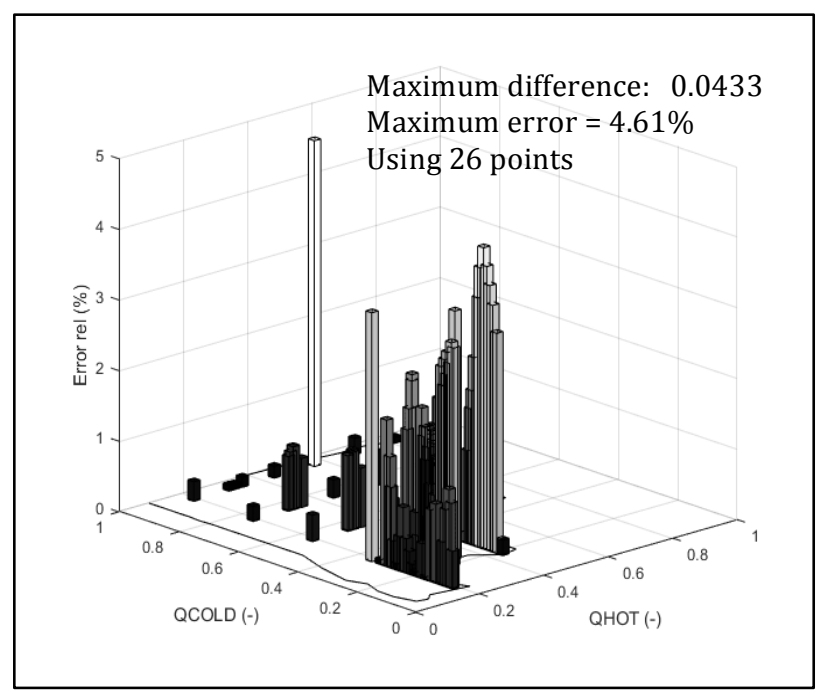

Fig. 14. Minimum number of points to be tested and the error distribution obtained with 26 points and fit function. 
This research has been financed and supported by Research project (H2020-CS2-CFP04-2016-02 CleanSky project), ref. 755517.

\section{Conclusions}

A calibration method for numerical simulation models has been presented. This proposal is based on a look-up table correction strategy, which requires an original look-up table (information previously known) and a set of experimental data (new information). An optimization procedure to obtain the set of data, which is based on the test number minimization according detailed error comparison against existing previous data, is described. The calibration and optimization methodology has been applied on a heat exchanger component and results are shown. An automatic tool has been implemented to define the perimeter operating range, the location of the points into the contour and the minimum number of points required to find a good correction of the variable studied (e.g.: effectiveness in a HX). The results obtained for the HX are presented as an example. However, this tool can be applied on the different HVAC\&R components.

\section{References}

1. E. B. Ondes, I. Bayezit, I. Poergye, and A. Hafsi, Model-based 2-D look-up table calibration tool development, 11th Asian Control Conference (ASCC), IEEE Xplore, Gold Coast Convention Centre, Australia pp.17-20, December (2017)

2. S. Evangelista, G. Giovinco, S. Kocaman, A multiparameter calibration method for the numerical simulation of morphodynamic problems, J. Hydrol. Hydromech., Vol 65, 2, pp.175-18, (2017).

3. X. Lü, T. Lu, M. Viljanen, Calibrating numerical model by neural networks: A case study for the simulation of the indoor temperature of a building, Energy Procedia, Vol. 75, pp.366 - 1372, (2015).

4. G. E. Fasshauer, Meshfree Approximation Methods with MATLAB, Interdisciplinary Mathematical Sciences: Vol. 6, (2007), Illinois Institute of Technology, USA.

5. D. H. Mclain, Drawing contours from arbitrary data points, The Computer Journal, Vol. 17, pp.318-324, (1974).

6. MathWork, MATLAB - Mathematics - user Guide, (2018). 